\title{
Multiple Oral Dosing Pharmacokinetics of Standardized Extract of Centella asiatica ECa 233 and Its Inductive Effect on Efflux Transporters in Rats
}

\section{(ㄷ) (1) $($ ) $\Theta$}

Authors

Tosapol Anukunwithaya ${ }^{1}$, Mayuree H. Tantisira ${ }^{2,3}$, Tsutomu Shimada4, 5, Yoshimichi Sai ${ }^{4,5}$, Phisit Khemawoot ${ }^{1,2}$

\section{Affiliations}

1 Department of Pharmacology and Physiology, Faculty of Pharmaceutical Sciences, Chulalongkorn University, Bangkok, Thailand

2 Chulalongkorn University Drug and Health Products Innovation Promotion Center, Faculty of Pharmaceutical Sciences, Chulalongkorn University, Bangkok, Thailand

3 Faculty of Pharmaceutical Sciences, Burapha University, Chonburi, Thailand

4 Department of Clinical Pharmacokinetics, Graduate School of Medical Sciences, Kanazawa University, Takara-machi, Kanazawa, Japan

5 Department of Hospital Pharmacy, University Hospital, Kanazawa University, Takara-machi, Kanazawa, Japan

\section{Key words}

Centella asiatica, Apiaceae, ECa 233, madecassoside, asiaticoside, pharmacokinetics

received 14.03 .2017

revised 15.05.2017

accepted 06.06.2017

Bibliography

DOI https://doi.org/10.1055/s-0043-114669

Planta Med Int Open 2017; 4: e66-e73

(c) Georg Thieme Verlag KG Stuttgart · New York ISSN 2509-9264

\section{Correspondence}

Phisit Khemawoot, Ph.D.

Department of Pharmacology and Physiology

Faculty of Pharmaceutical Sciences

Chulalongkorn University

Bangkok 10330

Thailand

Tel.: +66/2/218 8343, Fax: + 66/2/218 8340

phisit.k@chula.ac.th 丹upporting information available online at http://www. thieme-connect.de/products

\section{ABSTRACT}

ECa 233 is a standardized extract of Centella asiatica, characterized as a white powder containing triterpenoid glycosides not less than $80 \%$ with a ratio of madecassoside to asiaticoside of $1.5 \pm 0.5: 1$. Although pharmacological and toxicological profiles of ECa 233 have been successively reported, the pharmacokinetic data needed for further therapeutic development are not fully elucidated. This study aimed to investigate the pharmacokinetics of multiple oral dosing of ECa 233 at $100 \mathrm{mg} / \mathrm{kg} /$ day for 7 days in rats. Plasma, tissues, urine, and feces were collected from 0 to $24 \mathrm{~h}$ after dosing on days 1 and 7 . The concentrations of asiaticoside, madecassoside, asiatic acid, and madecassic acid were simultaneously analyzed by liquid chromatography-tandem mass spectrometry. No significant change was observed in physical and blood biochemical parameters of the animals treated with ECa 233 for 7 days. The maximum plasma concentration and area under the curve at day 7 of madecassoside and asiaticoside decreased by $70-80 \%$ from day 1 . However, both triterpenoid glycosides were extensively distributed and accumulated, resulting in significantly higher concentrations at pharmacologically relevant organs. Madecasssic acid and asiatic acid are major metabolites mainly found in and excreted via feces. Moreover, multiple dosing of ECa 233 increased mRNA expression of $A b c b 1 a$ and $A b c c 2$ in the small intestine by approximately 2 - to 3 -fold. This is the first study to identify an inductive effect of a standardized extract of $C$. asiatica after multiple oral dosing in rats. Potential drugherb interactions when ECa 233 is coadministered with $A b c b 1 a$ and $A b c c 2$ substrates calls for further investigations. 


\section{Abbreviations}

$\begin{array}{ll}\text { ALT } & \text { alanine aminotransferase } \\ \text { AST } & \text { aspartate aminotransferase } \\ \text { AUC }_{(0-\mathrm{t})} & \begin{array}{l}\text { area under the curve from time } 0 \text { to the observed time } \\ \mathrm{C}_{\max }\end{array} \\ \text { ECa } 233 & \text { standardized extract of Centella asiatica } \\ \text { IS } & \text { internal standard } \\ \text { MRP2 } & \text { multidrug-resistant protein } 2 \\ \text { NSS } & \text { normal saline solution } \\ \text { PGP } & \text { P-glycoprotein } \\ \text { qPCR } & \text { real-time polymerase chain reaction } \\ T_{\max } & \text { time to reach maximum plasma concentration } \\ \text { XlogP } & \text { partition coefficient }\end{array}$

\section{Introduction}

Centella asiatica (L.) Urb. (Asiatic pennywort, Gotu kola, or Bua-bok) belongs to the Apiaceae family and is commonly found in tropical areas of Southeast Asia, India, South Africa, South America, and Eastern Europe [1-3]. The plant has been used as traditional medicine for a long time and, in line with this, supporting pharmacological effects have been well documented in a number of preclinical studies, suggesting the potential for further development of $C$. asiatica into a phytopharmaceutical product [4-11]. Considerable fluctuations in the chemical composition of the plant from different origins have resulted in inconsistencies of bioactive constituents of the plant extract, which poses a major problem for its application as a natural medicine. To overcome such a problem, we prepared ECa 233 which is a standardized extract of C. asiatica [12] defined as a white to off-white powder containing triterpenoid glycosides not less than $80 \%$ with a ratio of madecassoside to asiaticoside of 1.5 $\pm 0.5: 1$ ( $\triangleright$ Fig. 1) [13, 14].

ECa 233 has demonstrated various pharmacological effects such as the acceleration of second degree burn wound healing in Wistar rats [15], as well as a reduction in pain, ulcer size, and erythema score of minor recurrent aphthous ulcerations in human subjects [16]. In the central nervous system, the anxiolytic effects of $100 \mathrm{mg} / \mathrm{kg}$ ECa 233, given orally, have been shown in mouse models of acute and chronic stress [14]. ECa 233 was also found to ameliorate learning and memory deficits induced by intracerebroventricular injection of amyloid peptide in a mouse model [17]. The extract also has a neuritogenic effect on human neuroblastoma cells via the upregulation of activated ERK $1 / 2$ and Akt [18]. An oral dose up to $10 \mathrm{~g} / \mathrm{kg}$ has modest toxicity in both acute and subchronic tests in rodents [19]. Taken together, ECa 233 may be a good phytomedicine candidate for the treatment of some neurological disorders. However, the pharmacokinetic data needed for appropriate dosing are rather scant.

Recently, a single-dose pharmacokinetic study of orally given ECa 233 was conducted in rats [20]. It was found that, after a single oral doses of ECa 233 at 50, 100, and $200 \mathrm{mg} / \mathrm{kg}$, madecassoside and asiaticoside were rapidly absorbed, reaching their respective $C_{\max }$ (madecassoside $1654 \pm 884,5664 \pm 3947$, and $9020 \pm 5744 \mu \mathrm{g} / \mathrm{L}$; asiaticoside $318 \pm 192,1283 \pm 1089$, and $4028 \pm 3157 \mu \mathrm{g} / \mathrm{L}$ ) within 5-15 min. Both compounds were distributed into the brain and other tissues and were eliminated mainly as madecassic acid and asiatic acid via feces [21]. The present study

$$
\text { (c) }
$$

- Fig. 1 Structures of two triterpenoid glycosides, madecassoside $(\mathrm{R}=\mathrm{OH})$ and asiaticoside $(\mathrm{R}=\mathrm{H})$, which are the major components of ECa 233.

- Table 1 Physical and biochemical profiles of rats pre- and post-treatment with oral dosing of ECa $233(100 \mathrm{mg} / \mathrm{kg} /$ day) for 7 consecutive days.

\begin{tabular}{|l|c|c|}
\hline $\begin{array}{l}\text { Biochemical } \\
\text { parameters }\end{array}$ & $\begin{array}{c}\text { Pretreatment at } \\
\text { day } \mathbf{1}\end{array}$ & $\begin{array}{c}\text { Post-treatment } \\
\text { at day 7 }\end{array}$ \\
\hline Physical appearance & Normal & Normal \\
\hline Creatinine $(\mathrm{mg} / \mathrm{dL})$ & $0.21 \pm 0.03$ & $0.22 \pm 0.04$ \\
\hline $\begin{array}{l}\text { Aspartate transaminase } \\
(\mathrm{U} / \mathrm{L})\end{array}$ & $61.56 \pm 11.45$ & $74.11 \pm 35.94$ \\
\hline $\begin{array}{l}\text { Alanine transaminase } \\
(\mathrm{U} / \mathrm{L})\end{array}$ & $18.67 \pm 7.53$ & $22.00 \pm 7.27$ \\
\hline
\end{tabular}

Data are shown as the mean \pm S.D. $(n=9) .{ }^{*} \mathrm{P}<0.05$ pretreatment on day 1 vs. post-treatment on day 7

further examines the single and multiple dosing pharmacokinetic behavior of ECa 233 at its effective dose for the treatment of chronic neurological conditions ( $100 \mathrm{mg} / \mathrm{kg}$ daily) in Wistar rats. The tissue distribution of the major components of ECa 233, namely madecassoside and asiaticoside, into pharmacologically related organs was also conducted to determine their steady state levels after multiple oral dosing for 7 days. Determination of triterpenoid glycosides and triterpenic acid metabolites was performed by LC-MS/ MS. In addition, the mRNA expression of efflux transporters in the liver and small intestine was determined by qPCR in order to access their effects on the pharmacokinetic behaviors of madecassoside and asiaticoside after multiple oral dosing of ECa 233. The data obtained from this study will help with determining the appropriate dose of ECa 233 for long-term use and highlights possible drugherb interactions of ECa 233.

\section{Results}

Rats receiving $100 \mathrm{mg} / \mathrm{kg} /$ day of oral ECa 233 for 7 days showed no significant physical changes or abnormalities. AST and ALT levels at post-treatment ( $24 \mathrm{~h}$ on day 7 ) did not change significantly from those of pretreatment ( 0 h on day 1 ), indicating marginal effects of ECa 233 on liver functions. Creatinine levels, reflecting kidney status, showed no significant changes after multiple oral dosing for 7 days. All biochemical parameters were within the normal ranges of healthy Wistar rats ( $\triangleright$ Table $\mathbf{1}$ ).

Plasma concentration-time profiles of madecassoside and asiaticoside, the major components of ECa 233, were constructed on 
days 1 and 7 ( $\triangleright$ Fig. 2), and the pharmacokinetic parameters were determined ( $\triangleright$ Table 2). Multiple oral doses of ECa 233 (100 mg/ $\mathrm{kg}$ ) for one week resulted in a significant decrease in the $C_{\max }$ and $\mathrm{AUC}_{(0-24)}$ of madecassoside and asiaticoside compared with the single oral dose on day $1(p<0.05) . C_{\max }$ decreased by 83.4 and $83.6 \%$ and $\mathrm{AUC}_{(0-24)}$ decreased by 78.2 and $72.2 \%$ for madecassoside and asiaticoside, respectively. $T_{\max }$ at day 7 was slightly delayed, but did not differ significantly from that observed on day 1 .

Madecassoside and asiaticoside showed good tissue distribution after oral administration of $100 \mathrm{mg} / \mathrm{kg}$ ECa 233 for 7 consecutive days ( $\triangleright$ Table 3 ). Both compounds reached the brain, skin, spleen, liver, kidney, and stomach in similar distribution patterns with the $\mathrm{AUC}_{(0-4)}$ up to $44.5 \mathrm{\mu g} \cdot \mathrm{h} / \mathrm{g}$ of tissue. Interestingly, the $\mathrm{AUC}_{(0-4)}$ of all tissues, except the stomach, after ECa 233 dosing on day 7 was sig-

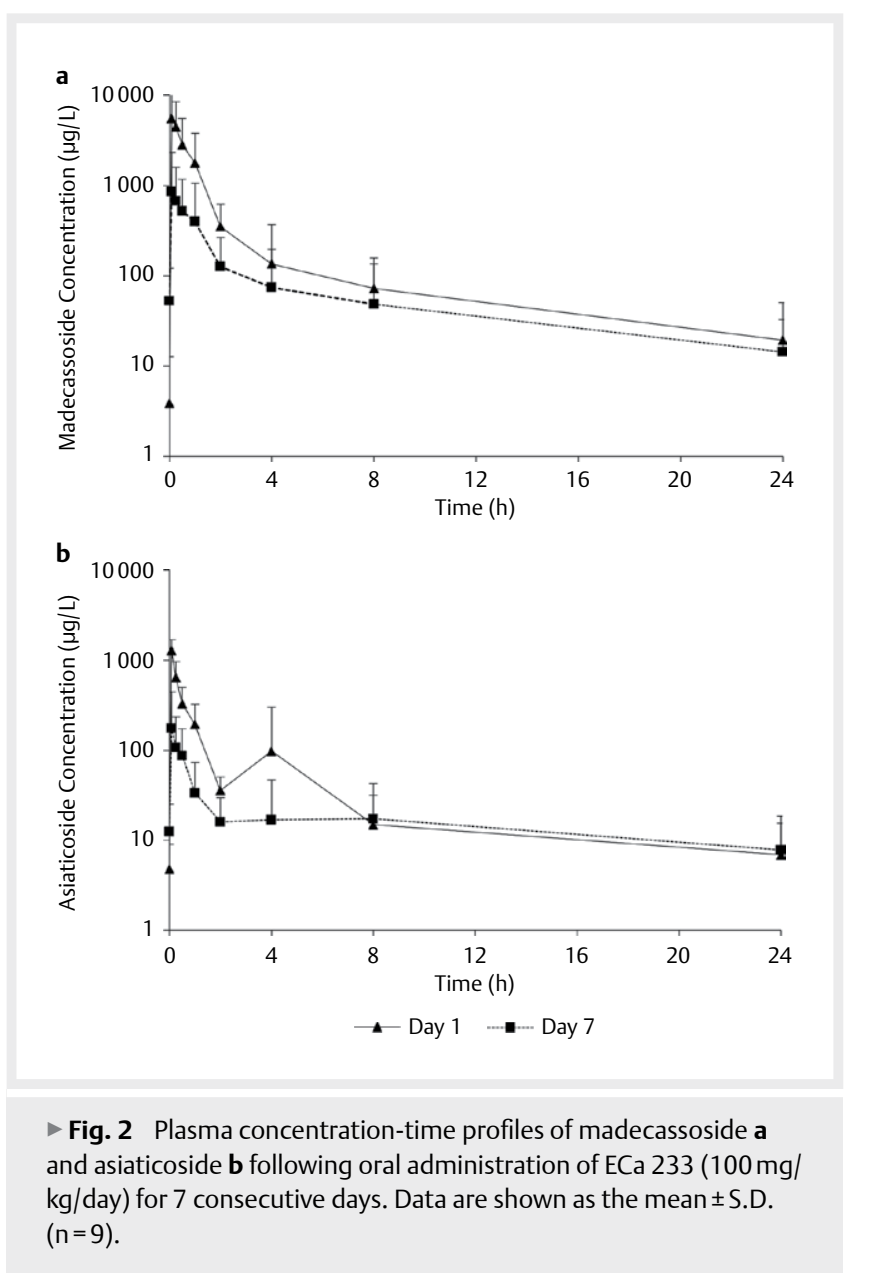

nificantly higher than that after single oral dosing (day 1 ) in which madecassoside and asiaticoside in the brain were increased by approximately 4- and 6-fold, respectively. Excretion of triterpenoid glycosides was observed within $24 \mathrm{~h}$ after dosing on days 1 and 7 ( $\triangleright$ Table 4). Madecassoside and asiaticoside were detected in negligible amounts in the urine. Madecassic acid and asiatic acid, the expected triterpenic acid metabolites of madecassoside and asiaticoside, which were found at negligible levels in plasma, were detected mainly in the feces and had higher percent recoveries than the parent compounds on days 1 and 7 .

The mRNA expression levels of $A b c b 1 a, A b c b 1 b, A b c c 2$, and $A b c g 2$ in the liver and small intestine were quantified ( $\triangleright$ Fig. 3 ). There were no notable changes in any of the observed efflux transporter mRNAs in the liver of most tested groups. The mRNA expression of $A b c b 1 a$ and $A b c c 2$ was significantly upregulated in the small intestine. There was about a 2-fold increase in $A b c b 1 a$ expression in the duodenum and jejunum, and a 2- to 4-fold increase of $A b c c 2$ mRNA expression was clearly observed throughout the small intestine of the animals receiving multiple doses of ECa 233 for 7 days.

\section{Discussion}

ECa 233 is a standardized extract of $C$. asiatica that consistently contains designated levels of madecassoside and asiaticoside [12]. The pharmacological effects of ECa 233 in decreasing anxiety [14] and ameliorating learning and memory deficits indicate the therapeutic potential of this extract $[17,18]$. Development of ECa 233 as an alternative medicine for the treatment of some central nervous system disorders requires pharmacokinetic data following longterm administration. In this study, the multiple-dose pharmacokinetics of $100 \mathrm{mg} / \mathrm{kg}$ given orally for 7 days were examined in adult male Wistar rats. No animals showed significant changes in physical appearance or biochemical parameters, indicating normal kidney and liver function. These results are consistent with the good safety profiles of ECa 233 in previous acute and subchronic toxicity studies [19].

The pharmacokinetic parameters of madecassoside and asiaticoside were determined from the plasma concentration-time profiles on days 1 and 7 using non-compartmental analysis. The significant $80 \%$ decreases in $C_{\max }$ and $A U C_{(0-24)}$ of madecassoside and asiaticoside after 7 consecutive days of ECa 233 treatment indicate the possibility that the extract might have an inductive effect on efflux transporters or on drug metabolizing enzymes, as previously demonstrated by other herbal products [22,23]. Madecassoside has been found to be a substrate for efflux transporters such as PGP and MRP2 [24]. Accordingly, our qPCR results did demonstrate in-

- Table 2 Pharmacokinetic parameters of madecassoside and asiaticoside after oral dosing of ECa 233 (100 mg/kg/day) on days 1 and 7 .

\begin{tabular}{|c|c|c|c|c|}
\hline \multirow{2}{*}{$\begin{array}{l}\text { Pharmacokinetic } \\
\text { parameters }\end{array}$} & \multicolumn{2}{|c|}{ Madecassoside } & \multicolumn{2}{|c|}{ Asiaticoside } \\
\hline & Day 1 & Day 7 & Day 1 & Day 7 \\
\hline$C_{\max }(\mu \mathrm{g} / \mathrm{L})$ & $5713.00 \pm 5069.38$ & $948.03 \pm 1449.37^{*}$ & $1281.60 \pm 407.48$ & $209.62 \pm 250.74^{*}$ \\
\hline $\mathrm{T}_{\max }(\mathrm{h})$ & $0.17 \pm 0.14$ & $1.30 \pm 2.72$ & $0.08 \pm 0.00$ & $1.50 \pm 3.19$ \\
\hline $\operatorname{AUC}_{(0-24)}(\mu \mathrm{g} \cdot \mathrm{h} / \mathrm{L})$ & $7361.88 \pm 4195.27$ & $1604.40 \pm 2066.20 *$ & $1104.98 \pm 713.29$ & $306.78 \pm 234.37$ * \\
\hline $\begin{array}{l}\text { Normalized } \mathrm{AUC}_{(0-24)} \\
(\mu \mathrm{g} \cdot \mathrm{h} \cdot \mathrm{kg} / \mathrm{L} \cdot \mathrm{mg})\end{array}$ & $138.64 \pm 79.01$ & $30.21 \pm 38.91^{*}$ & $34.21 \pm 22.08$ & $9.50 \pm 7.26^{*}$ \\
\hline
\end{tabular}


- Table 3 Area under the curve of madecassoside and asiaticoside in internal organs from time $0-4 \mathrm{~h}$ after oral dosing of ECa 233 (100 mg/kg/day) on days 1 and 7.

\begin{tabular}{|c|c|c|c|}
\hline \multirow{2}{*}{ Compounds } & \multirow{2}{*}{ Organs } & \multicolumn{2}{|c|}{$\operatorname{AUC}_{(0-4)}(\mathrm{ng} \cdot \mathrm{h} / \mathrm{g}$ of tissue) } \\
\hline & & Day 1 & Day 7 \\
\hline \multirow[t]{6}{*}{ Madecassoside } & Skin & $613 \pm 97$ & $2019 \pm 947^{*}$ \\
\hline & Spleen & $206 \pm 97$ & $4048 \pm 4104^{*}$ \\
\hline & Brain & $84 \pm 32$ & $309 \pm 194^{*}$ \\
\hline & Stomach & $43538 \pm 11383$ & $44496 \pm 25460$ \\
\hline & Kidney & $1602 \pm 2110$ & $21814 \pm 18301^{*}$ \\
\hline & Liver & $1524 \pm 2191$ & $6597 \pm 3967^{*}$ \\
\hline \multirow[t]{6}{*}{ Asiaticoside } & Skin & $116 \pm 16$ & $1410 \pm 970^{*}$ \\
\hline & Spleen & $35 \pm 28$ & $2847 \pm 2796^{*}$ \\
\hline & Brain & $20 \pm 3$ & $120 \pm 97^{*}$ \\
\hline & Stomach & $22372 \pm 4599$ & $35313 \pm 21714$ \\
\hline & Kidney & $305 \pm 419$ & $18105 \pm 15629^{*}$ \\
\hline & Liver & $343 \pm 529$ & $4119 \pm 2828^{*}$ \\
\hline
\end{tabular}

- Table 4 Percent recovery of triterpenoid glycosides and triterpenic acid metabolites for $0-24 \mathrm{~h}$ after oral dosing of ECa 233 (100 mg/kg/day) on days 1 and 7.

\begin{tabular}{|l|c|c|c|c|c|c|}
\hline \multirow{2}{*}{ Percent recovery } & \multicolumn{3}{|c|}{ Day 1} & \multicolumn{2}{c|}{ Day 7} \\
\cline { 2 - 7 } & Urine & Feces & Total & Urine & Feces & $0.09 \pm 0.18$ \\
\hline Madecassoside & $0.01 \pm 0.01$ & $9.57 \pm 20.14$ & $9.58 \pm 20.13$ & $0.01 \pm 0.02$ & $0.11 \pm 0.18$ \\
\hline Asiaticoside & $0.00 \pm 0.00$ & $2.61 \pm 4.70$ & $2.61 \pm 4.70$ & $0.00 \pm 0.00$ & $0.10 \pm 0.20$ & $0.10 \pm 0.20$ \\
\hline Madecassic acid & $0.00 \pm 0.00$ & $17.43 \pm 8.50$ & $17.43 \pm 8.50$ & $0.00 \pm 0.00$ & $21.34 \pm 12.93$ & $21.34 \pm 12.93$ \\
\hline Asiatic acid & $0.00 \pm 0.00$ & $10.95 \pm 5.23$ & $10.95 \pm 5.23$ & $0.00 \pm 0.00$ & $5.40 \pm 2.92$ & $5.40 \pm 2.92 *$ \\
\hline \multicolumn{2}{|l}{ Data are shown as the mean \pm S.D. $(n=9) .{ }^{*}$ P $<0.05$ total percent recovery on day 1 vs. day 7} \\
\hline
\end{tabular}

creasing mRNA levels of $A b c b 1 a$ and $A b c c 2$, which are responsible for PGP and MRP2 expression. These two transporters could be upregulated in the small intestine, especially the duodenum and jejunum where most drug absorption takes place. Together with our previous findings, ECa 233 neither altered the total CYP content nor the CYP1A1, CYP1A2, CYP2B1/2B2, CYP2E1, and CYP3A activities after 90 days of treatment [25]. It is suggestive that upregulation of the efflux transporters, PGP and MRP2, might underlie the decreases in the $C_{\max }$ and $\mathrm{AUC}_{(0-24)}$ of madecassoside and possibly asiaticoside as well. On day 1 of oral dosing with ECa 233, the $T_{\max }$ of madecassoside and asiaticoside was found to be $0.08-0.17 \mathrm{~h}$ and tended to be delayed after multiple dosing of ECa 233. A small second peak $4 \mathrm{~h}$ after dosing could possibly be accounted for by the enterohepatic circulation as proposed for pure madecassoside in a linked-rat model [24].

The tissue distribution study showed that madecassoside and asiaticoside reached various organs such as the skin, stomach, and especially the brain in which ECa 233 apparently exerts its pharmacological effects. High amounts of both triterpenoid glycosides in brain tissue is key evidence to confirm that madecassoside and asiaticoside are the bioactive compounds responsible for the pharmacological effects of ECa 233, as previously stated in a single dosing pharmacokinetic study [20]. In the present study, both triterpenoid glycosides appeared to be rapidly absorbed from the intestine into the circulatory system and extensively distributed into the internal organs after multiple oral dosing. Accumulation of these two glycosides in the target organs was apparent and resulted in a significantly higher tissue $\mathrm{AUC}_{(0-4)}$ of madecassoside and asiaticoside on day 7 , while their respective plasma concentrations were decreasing. Hence, our study suggests that it is best to determine the tissue levels of the active constituents of the herbal extract in concurrent with their respective plasma concentration-time profiles in multiple dosing pharmacokinetic studies.

Madecassic acid and asiatic acid were initially suggested to be active triterpenic metabolites [26] formed in vivo by esterase hydrolytic cleavage or acid hydrolysis of the sugar moiety from the parent triterpenoid glycosides [27] in a stepwise process [24]. The levels of these metabolites were minimal in plasma and tissue samples, but they were abundantly found in feces. The calculated percent recoveries within $24 \mathrm{~h}$ post-treatment on days 1 and 7 were consistent with our previous studies showing that ECa 233 was primarily excreted via feces and mainly as madecassic acid and asiatic acid [20,21]. This result could imply that the parent triterpenoid glycosides underwent a hydrolytic reaction to remove the sugar moiety and were then metabolized into the acid metabolites in the gastrointestinal tract. A relatively larger amount of triterpenic acid metabolites, in comparison with their respective parent triterpenoid glycosides, were found in the feces on days 1 and 7. A small amount of triterpenic acid metabolites was excreted via the urinary system, consistent with their lipophilic properties (madecassic acid: 

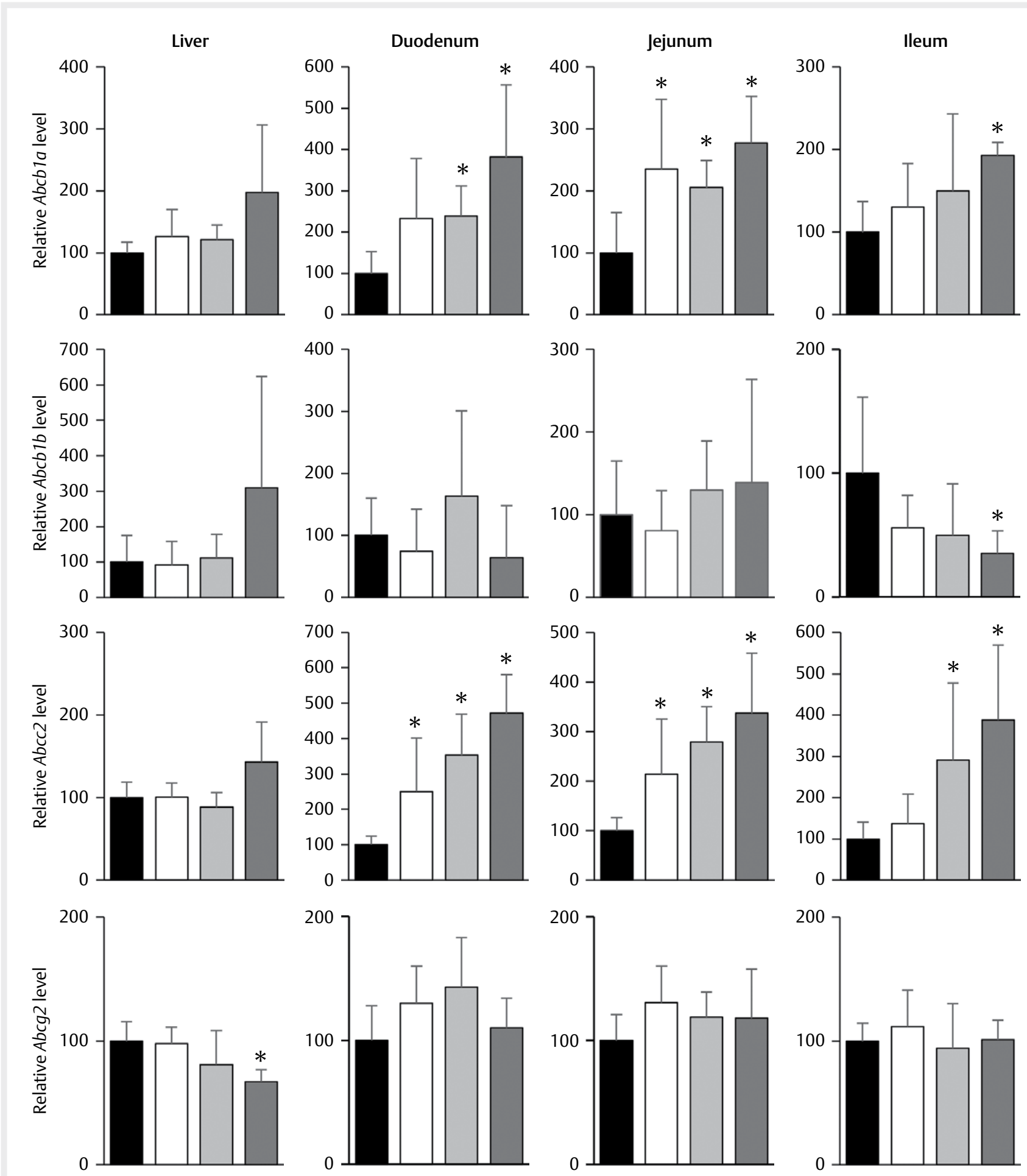

Control $\square 50 \mathrm{mg} / \mathrm{kg}$ ECa $233 \square 100 \mathrm{mg} / \mathrm{kg}$ ECa $233 \square 250 \mathrm{mg} / \mathrm{kg}$ Rifampicin

- Fig. 3 Relative mRNA expression level (\% of control) of $A b c b 1 a, A b c b 1 b, A b c c 2$, and $A b c g 2$ in the liver and small intestine after multiple oral dosing for 7 consecutive days. Data are shown as the mean \pm S.D. $(n=6) .{ }^{*} P<0.05$ vs. control group. 
XlogP 4.4, and asiatic acid: XlogP 5.7). Therefore, both of compounds were excreted mainly by the hepatobiliary system, rather than the urinary system.

In conclusion, a significant accumulation of madecassoside and asiaticoside in the tissue compartments was apparent despite an $80 \%$ decrease in their respective plasma concentrations at day 7 . This decrease in plasma triterpenoid glycosides could be accounted for by the inductive effect of ECa 233 on intestinal Abcb1a and Abcc2 mRNA expression. The information obtained in this study suggests that no dosage adjustment is required for long-term use of ECa 233, but calls attention to the possibility of drug-herb interactions when ECa 233 is coadministered long term with conventional PGP and MRP2 substrate drugs.

\section{Materials and Methods}

\section{Chemicals}

ECa 233 was kindly supplied by Siam Herbal Innovation Co., Ltd. (batch number MRA1214004) as a white powder containing $53.1 \%$ madecassoside and $32.3 \%$ asiaticoside. Analytical standards of asiaticoside (>98.5\%), madecassoside (>95.0\%), and asiatic acid ( $>95.0 \%$ ) were purchased from Sigma-Aldrich Corp. Rifampicin (>90.0\%), madecassic acid (>98.9\%), and glycyrrhetinic acid (>99.0\%) were obtained from Wako Pure Chemical Industries.

\section{Animals}

Male Wistar rats aged 12 weeks old were obtained from the $\mathrm{Na}$ tional Laboratory Animal Center, Mahidol University. They were housed with free access to food ad libitum under a controlled temperature and 12-h light-dark cycle prior to multiple dosing experiments. Rats with a weight of 400 to $600 \mathrm{~g}$ were placed in metabolic cages and fasted for $12 \mathrm{~h}$ with free access to water before a daily dosing of ECa 233, rifampicin, or vehicle (20\% DMSO/NSS). The animal experiments were conducted according to a protocol approved by the Institutional Animal Care and Use Committee of the Faculty of Pharmaceutical Sciences, Chulalongkorn University, Bangkok, Thailand (approval number 13-33-007 and approval date: March 4, 2013).

\section{Pharmacokinetic experiments}

Rats received a daily oral administration of freshly prepared ECa $233(50 \mathrm{mg} / \mathrm{mL}$ in $20 \%$ DMSO/NSS) at a dose of $100 \mathrm{mg} / \mathrm{kg}$ for 7 days. Blood $(400 \mu \mathrm{L})$ was collected via the lateral tail vein into preheparinized tubes on days 1 and 7 at 0, 0.083, 0.25, 0.5, 1, 2, 4, 8, and $24 \mathrm{~h}$ after dosing. Blood samples were centrifuged at $1500 \times \mathrm{g}$ for $10 \mathrm{~min}$ to acquire approximately $200 \mu \mathrm{L}$ of plasma. Urine and feces were collected separately from metabolic cages on days 1 and 7 for $0-24 \mathrm{~h}$ after dosing. Urine volume and feces weight were recorded for the calculation of percent recovery. On day 7 , some rats were euthanized at 1,2 and $4 \mathrm{~h}$ after oral dosing to collect the brain, liver, kidney, spleen, stomach, and skin. Tissue samples were washed in normal saline to remove blood. All rats were anesthetized with isoflurane to reduce pain and injury during drug administration and sample collection. All biological samples were kept at $-20^{\circ} \mathrm{C}$ until analysis. Plasma samples at $0 \mathrm{~h}$ on day 1 and $24 \mathrm{~h}$ on day 7 were also used for evaluation of creatinine, AST, and ALT levels to examine the effects of ECa 233 on kidney and liver functions.

\section{Sample preparation}

Methanol was used as a precipitating agent to prepare all biological samples by protein precipitation. Plasma and urine samples were thawed at room temperature, and $50 \mu \mathrm{L}$ of each sample were mixed with $200 \mu \mathrm{L}$ of methanol containing $10 \mathrm{ng}$ of glycyrrhetinic acid (IS). The mixture was centrifuged at $5000 \times \mathrm{g}$ for $10 \mathrm{~min}$, and $10 \mu \mathrm{L}$ of supernatants were analyzed by LC-MS/MS using optimized conditions and parameters. Approximately $50 \mathrm{mg}$ of feces or tissue were combined with $200 \mu \mathrm{L}$ of methanol containing $10 \mathrm{ng}$ of IS, homogenized in an ice bath, and then processed in the same manner as the plasma and urine samples. Blank matrices were used to dilute some samples before protein precipitation if the concentration of analytes exceeded the linearity range of detection in LC-MS/MS.

\section{LC-MS/MS analysis}

LC-MS/MS analysis was carried out using methods previously mentioned in a pharmacokinetic study of a single oral dosing of ECa 233 [20]. In brief, an LC-MS/MS system comprising of an Eksigent Ekspert UPLC 100 liquid chromatograph equipped with a QTRAP 6500 mass spectrometer controlled by Analyst software version 1.6 (AB Sciex Pte. Ltd.) was used for analytical procedures. The stationary phase was a Synergi $4 \mu \mathrm{m}$ Fusion-RP C18 column $50 \times 2 \mathrm{~mm}$ (Phenomenex, Inc.) with a $40^{\circ} \mathrm{C}$ oven temperature. With the optimized LC-MS/MS system, the retention times of madecassoside, asiaticoside, madecassic acid, asiatic acid, and glycyrrhetinic acid were $1.79,1.82,1.93,1.99$ and $2.12 \mathrm{~min}$, respectively. The calculated percent recoveries were $78-93 \%$ for all analytes. Calibration curves of triterpenoid glycosides and triterpenic acids showed good linearity with $R^{2}>0.99$ for $0.5-300 \mu \mathrm{g} / \mathrm{L}$. The lower limit of detection was $0.1-0.5 \mu \mathrm{g} / \mathrm{L}$, and intraday and inter-day precision and accuracy were within $\pm 10 \%$.

\section{Quantitative real-time PCR}

Rats were divided into four groups and orally treated for 7 days as vehicle (negative control), $50 \mathrm{mg} / \mathrm{kg}$ ECa 233 (low dose), $100 \mathrm{mg} /$ $\mathrm{kg}$ ECa 233 (high dose), and $250 \mathrm{mg} / \mathrm{kg}$ rifampicin (positive control). Subsequently, they were euthanized to collect liver and intestinal mucosal cells from the duodenum, jejunum, and ileum parts of the small intestine. Total RNA was then isolated from tissue homogenates using a Gen Elute Mammalian Total RNA Miniprep Kit (Sigma-Aldrich Corp.) according to the company instructions. The cDNA was consequently prepared by reverse transcription of each RNA sample with a High Capacity cDNA Reverse Transcription Kit (Applied Biosystems). Quantitative real-time PCR was performed on an Mx3000P Real-Time QPCR System (Agilent Technologies Inc.). The following primers were used for amplification of rat Gapdh: forward 5'-CTG TGG TCA TGA GCC CCT CC-3' and reverse 5'-CGC TGG TGC TGA GTA TGT CG-3', Abcb1a: forward 5'-TGA ACT GTG ACC ATG CGA GAT GTT AAA TA-3' and reverse 5'-GTC TCT GAA GAC TCT AAA ATG GAC TAA ATG-3', Abcb 1b: forward 5'-CCA GGA GAG AAG ACT TAG TTC G-3' and reverse 5'-GGC AAA CAC TGG TTG TAT GCA C-3', Abcc2: forward 5'-TTC ACG GGC ACA TCA CCA-3' and reverse 5'ATT CGG ACC CAA ACA GGA TG-3', Abcg2: forward 5'-GTT TGG ACT 
CAA GCA CAG CA-3' and reverse 5'-TGA GTT TCC CAG AAG CCA GT-3', respectively. The qPCR conditions were one cycle at $95^{\circ} \mathrm{C}$ for $1 \mathrm{~min}$, followed by 40 cycles of $95^{\circ} \mathrm{C}$ for $15 \mathrm{~s}, 55^{\circ} \mathrm{C}$ for $15 \mathrm{~s}$, and $72^{\circ} \mathrm{C}$ for $30 \mathrm{~s}$, and a last cycle of $95^{\circ} \mathrm{C}$ for $1 \mathrm{~min}, 55^{\circ} \mathrm{C}$ for $30 \mathrm{~s}$, and $95^{\circ} \mathrm{C}$ for $30 \mathrm{~s}$.

\section{Data analysis}

Biochemical parameters for the liver and kidney were analyzed by Student's t-test $(p<0.05)$ to determine significant differences between pretreatment ( $0 \mathrm{~h}$ on day 1 ) and post-treatment ( $24 \mathrm{~h}$ on day 7). Pharmacokinetic parameters of madecassoside and asiaticoside, including $C_{\max }, T_{\max }, A \cup C_{(0-24)}$, and normalized $A U C_{(0-24)}$, and tissue $\mathrm{AUC}_{(0-4)}$ were reported. The calculated concentrations of madecassoside, asiaticoside, madecassic acid, and asiatic acid in urine and feces were used to determine percent recoveries using the total drug found in urine and feces divided by the administered dose based on a molar basis. Statistical differences in pharmacokinetic parameters, tissue $\mathrm{AUC}_{(0-4)}$, and percent recoveries between day 1 and day 7 were analyzed using Student's t-test $(p<0.05)$. The expression level of each mRNA was normalized by the expression of Gapdh as an internal control, and reported as \% of the control group. Student's t-test $(p<0.05)$ was also used to evaluate the statistical differences between the ECa 233 treated groups and the control group.

\section{Acknowledgements}

The authors are grateful for the scholarships from the Graduate School of Chulalongkorn University to commemorate the 72nd anniversary of His Majesty King Bhumibala Aduladeja, and the overseas research experience scholarship for graduate students. We also express our gratitude to the Faculty of Pharmaceutical Sciences, Chulalongkorn University for providing research funds for Dr. Phisit Khemawoot [Grant number, Phar2560-RG04].

\section{Conflict of Interest}

All authors declare no conflicts of interest.

\section{References}

[1] Jamil SS, Nizami Q, Salam M. Centella asiatica (Linn.) Urban: a review. Indian J Nat Prod Res 2007; 6: 158-170

[2] Krittapun W. Usefulness of Herbs. Department of Pharmacognosy, Faculty of Pharmacy, Mahidol University; 1996

[3] Bown D. Encyclopedia of herbs and their uses (Herb Society of America). New York: Dorling Kindersley; 1995

[4] Maquart FX, Chastang F, Simeon A, Birembaut P, Gillery P, Wegrowski Y. Triterpenes from Centella asiatica stimulate extracellular matrix accumulation in rat experimental wounds. Eur J Dermatol 1999; 9 : 289-296

[5] Shukla A, Rasik AM, Jain GK, Shankar R, Kulshrestha DK, Dhawan BN. In vitro and in vivo wound healing activity of asiaticoside isolated from Centella asiatica. J Ethnopharmacol 1999; 65: 1-11
[6] Veerendra Kumar MH, Gupta YK. Effect of Centella asiatica on cognition and oxidative stress in an intracerebroventricular streptozotocin model of Alzheimer's disease in rats. Clin Exp Pharmacol Physiol 2003; 30: 336-342

[7] Cheng CL, Koo MWL. Effects of Centella asiatica on ethanol induced gastric mucosal lesions in rats. Life Sci 2000; 67: 2647-2653

[8] Cesarone MR, Belcaro G, De Sanctis MT, Incandela L, Cacchio M, Bavera P, Ippolito E, Bucci M, Griffin M, Geroulakos G, Dugall M, Buccella S, Kleyweght S, Cacchio M. Effects of the total triterpenic fraction of Centella asiatica in venous hypertensive microangiopathy: a prospective, placebo-controlled, randomized trial. Angiology 2001; 52: S15-S18

[9] Punturee K, Wild CP, Vinitketkumneun U. Thai medicinal plants modulate nitric oxide and tumor necrosis factor- $\alpha$ in $J 774.2$ mouse macrophages. J Ethnopharmacol 2004; 95: 183-189

[10] James JT, Dubery IA. Pentacyclic triterpenoids from the medicinal herb, Centella asiatica (L.) Urban. Molecules 2009; 14: 3922-3941

[11] Guo JS, Cheng CL, Koo MWL. Inhibitory effects of Centella asiatica water extract and asiaticoside on inducible nitric oxide synthase during gastric ulcer healing in rats. Planta Med 2004; 70: 1150-1154

[12] Saifah E, Suttisri R, Patarapanich C, Laungchonlatan S, Tantisira MH, Tantisira B. Preparation methods of colorless mixture between madecassoside and asiaticoside from Centella asiatica. In:Department of Intellectual Property MoC. ed. $\mathrm{CO} 7 \mathrm{H}$ 1/00 ed.Bangkok, Thailand: Chulalongkorn University; 2009

[13] Tantisira MH. Bioactive standardized extract of Centella asiatica (ECa 233).In: The Eighth NRCT-JSPS Joint Seminar.Bangkok, Thailand: Chulalongkorn University Printing Press; 200925

[14] Wanasuntronwong A, Tantisira MH, Tantisira B, Watanabe H. Anxiolytic effects of standardized extract of Centella asiatica (ECa 233) after chronic immobilization stress in mice. J Ethnopharmacol 2012; 143 579-585

[15] Wannarat K, Tantisira MH, Tantisira B. Wound healing effects of a standardized extract of Centella asiatica ECa 233 on burn wound in rats. Thai J Pharmacol 2009; 31: 120-123

[16] Ruengprasertkit C, Hongprasong N, Tantisira B, Tantisira MH. Preliminary study of effects of a standardized extract of Centella asiatica ECa 233 on minor aphthous ulcers. Chulalongkorn Univ Dent ] 2010; 33: 131-142

[17] Kam-eg A, Tantisira B, Tantisira MH. Preliminary study on effects of a standardized extract of Centella asiatica, ECa 233, on deficit of learning and memory induced by an intracerebroventricular injection of $\beta$-amyloid peptide in mice. Thai J Pharmacol 2009; 31: 79-82

[18] Wanakhachornkrai O, Pongrakhananon V, Chunhacha P, Wanasuntronwong A, Vattanajun A, Tantisira B, Chanvorachote P, Tantisira M. Neuritogenic effect of standardized extract of Centella asiatica ECa 233 on human neuroblastoma cells. BMC Complement Altern Med 2013; $13: 204$

[19] Chivapat S, Chavalittumrong P, Tantisira MH. Acute and sub-chronic toxicity studies of a standardized extract of Centella asiatica ECa 233. Thai J Pharm Sci 2011; 35: 55-64

[20] Anukunwithaya T, Tantisira MH, Tantisira B, Khemawoot P. Pharmacokinetics of a standardized extract of Centella asiatica ECa 233 in rats. Planta Med 2017; 83: 710-717

[21] Hengjumrut P, Anukunwithaya T, Tantisira MH, Tantisira B, Khemawoot P. Comparative pharmacokinetics between madecassoside and asiaticoside presented in a standardised extract of Centella asiatica, ECa 233 and their respective pure compound given separately in rats. Xenobiotica advance online publication 8 January 2017 doi:10.1080/0 0498254.2016 .1273562 
[22] Tirona RG, Kim RB. Nuclear receptors and drug-drug interactions with prescription and herbal medicines. In: Xie W.editor. Nuclear receptors in drug metabolism. Hoboken, New Jersey: John Wiley \& Sons, Inc; 2008: 211-239

[23] Oga E, Sekine S, Shitara Y, Horie T. Pharmacokinetic herb-drug interactions: insight into mechanisms and consequences. Eur J Drug Metab Pharmacokinet 2016; 41: 93-108

[24] Leng DD, Han W], Rui Y, Dai Y, Xia YF. In vivo disposition and metabolism of madecassoside, a major bioactive constituent in Centella asiatica (L.) Urb. J Ethnopharmacol 2013; 150: 601-608

[25] Kulthong K, Tantisira MH, Niwattisaiwong N, Apipalakul K, Chevapat S, Lawanprasert S. Effects of the standard extract of Centella asiatica (ECa 233) on rat hepatic cytochrome P450. Thai J Pharm Sci 2009; 33: 91-100
[26] Krishnamurthy RG, Senut MC, Zemke D, Min J, Frenkel MB, Greenberg EJ, Yu SW, Ahn N, Goudreau J, Kassab M, Panickar KS, Majid A. Asiatic acid, a pentacyclic triterpene from Centella asiatica, is neuroprotective in a mouse model of focal cerebral ischemia. J Neurosci Res 2009; 87: 2541-2550

[27] Rush WR, Murray GR, Graham DJM. The comparative steady-state bioavailability of the active ingredients of madecassol. Eur J Drug Metab Pharmacokinet 1993; 18: 323-326 